\title{
A Tripeptide/Hexapeptide Anti-Aging Regimen that Targets Both Collagen and Elastin, and Improves both Physician and Subject Scoring of Facial Aesthetics
}

\author{
Nelson Aislyn M and Ortiz Arisa $\mathrm{E}^{*}$ \\ University of California, San Diego, Department of Dermatology, San Diego, CA , USA
}

*Corresponding author: Ortiz AE, Director, Laser and Cosmetic Dermatology, Assistant Clinical Professor, University of California, San Diego, Department of Dermatology, San Diego, CA, USA, Tel: (818) 744-3322; E-mail: arisaortiz@gmail.com

Received date: May 10, 2018; Accepted date: June 13, 2018; Published date: June 18, 2018

Copyright: @2018 Nelson, et al. This is an open-access article distributed under the terms of the Creative Commons Attribution License, which permits unrestricted use, distribution, and reproduction in any medium, provided the original author and source are credited.

\section{Abstract}

Background: A tripeptide/hexapeptide (TriHex) anti-aging regimen with a select blend of peptides and botanicals targeting skin aging by multiple actions was developed. The TriHex regimen was formulated to help clear the extracellular matrix (ECM) of degraded proteins and promote collagen and elastin production.

Aim: To assess the efficacy of an anti-aging regimen on moderate facial wrinkles and skin sagging.

Methods: Five subjects followed the anti-aging regimen for 12 weeks. Clinical photography, subject satisfaction surveys, subject global assessment, and physician grading were used to evaluate results.

Results: After subjects followed the anti-aging regimen for 12 weeks, statistically significant gains were noted in all physician-graded parameters. Compared to baseline, average percent improvements in skin fine lines (by $27.7 \%$ ), radiance (by $44.8 \%$ ), firmness (by $37.2 \%$ ), plumpness (by $31.6 \%$ ), sagginess, and wrinkles (both by $29.0 \%$ ) were observed. Similarly, the subject global assessment showed changes in most photoaging parameters, with positive effects noted as early as 8 weeks of use by a majority of subjects. By 12 weeks of use, all subjects either strongly agreed or agreed that the TriHex formulation improved their skin quality in each category measured by the survey.

Conclusions: Both subject and physician evaluations showed significant improvement in facial photoaging as early as 8 weeks after use of this tripeptide/hexapeptide anti-aging regimen. The anti-aging regimen improves multiple signs of photoaging using a synergistic blend of peptides and active botanicals.

Keywords: Anti-Aging; Botanicals; Collagen; Elastin; Extracellular matrix; Facial rejuvenation

\section{Introduction}

Photoaging is extrinsic damage to the skin caused by ultraviolet (UV) radiation [1]. The accumulation of this UV damage over time alters the dermal matrix along with the pigmentation of the skin [2]. The severity of photoaging is dependent on one's cumulative amount of sun exposure over time, i.e. outdoor activities, living in sunny climates. As the skin ages from both intrinsic (genetics) and extrinsic factors, dermal matrix components breakdown and their production is slowed [3]. The dermal matrix contains ECM proteins, collagen, elastin and other polypeptides and proteoglycans [1,3]. UV radiation can also trigger transfer of melanosomes and increased melanin production resulting in hyperpigmentation [4].

A unique serum with a combination of active peptides and botanicals (ALASTIN Restorative Skin Complex with TriHex Technology , ALASTIN Skincare, Carlsbad, CA) is designed to target much of the extrinsic damage that leads to skin aging. Select peptides and phosphatidylserine contained in the TriHex formulation clear the ECM of agglutinated protein fragments, stimulate intracellular protease and autophagy function, and initiate new collagen, elastin, decorin and dermatopontin replacement [5-7]. Hexapeptide 38 and ornithine are thought to act as peroxisome proliferator-activated receptor-gamma - PPAR $\gamma$ - coactivator 1 alpha (PGC1a) stimulators. PGCla can drive adipogenesis [8] and result in increased volume which may improve lines and wrinkles [9]. Other actives in the TriHex formulation, Dunaliela salina extract and niacinamide are antioxidants that can enhance pigmentation and skin quality [10-12]. The TriHex formulation's efficiency and its ability to penetrate the skin has been confirmed by in vivo biopsy analysis [6].

\section{Methods}

Five female subjects, 40-60 years old, with Fitzpatrick skin type 1-3 and signs of photoaging, were given an anti-aging regimen (Restorative Skin Complex with TriHex Technology", Daily Moisturizer, Broad Spectrum Sunscreen and Gentle Cleanser, ALASTIN Skincare, Carlsbad, CA). They were instructed to use the TriHex formulation, the moisturizer and the gentle cleanser twice a day and the sunscreen daily. Subjects did not use any other skin care products and did not undergo any laser or other cosmetic procedures during the treatment period. The trial was undertaken at the offices of Dr Ortiz 8899 University Center Ln, San Diego, CA 
Citation: Nelson AM, Ortiz AE (2018) A Tripeptide/Hexapeptide Anti-Aging Regimen that Targets Both Collagen and Elastin, and Improves both Physician and Subject Scoring of Facial Aesthetics. Dermatol Case Rep 3: 139.

Page 2 of 4

\section{Physician grading of efficacy}

Physician grading, performed by a board-certified physician, was performed at baseline and 8 and 12 weeks after initial use. Fine lines, radiance, skin firmness, skin plumpness, skin sagginess, and wrinkles were scored on a 10-point scale (Table 1). Statistics were performed using a pairedsample Student's $t$ test (accessed online at: http:// www.socscistatistics.com/tests/ttestdependent/Default.aspx [13]).

\begin{tabular}{|c|c|c|}
\hline Parameter & Location & Scoring scale $0-9^{a}$ \\
\hline Fine Lines & $\begin{array}{l}\text { Forehead, Crow's feet, Under eyes, cheek, nasolabial, marionette } \\
\text { lines }\end{array}$ & None to numerous deep, fine lines \\
\hline Radiance & Global face & Radiant/luminance appearance to dull/matte appearance \\
\hline Skin Firmness & Global face & Firm/dense appearance to soft/mushy appearance \\
\hline Skin plumpness & Global face & Raised/hydrated appearance to flat/dry appearance \\
\hline Skin sagginess & Jawline, cheek, and eye areas & None to visible/gravitational looseness \\
\hline Wrinkles & Global face & None to numerous deep wrinkles \\
\hline
\end{tabular}

Table 1: Scoring Scale for Physician Grading of Photoaging Parameters.

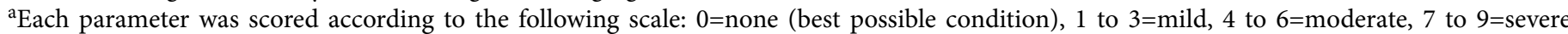
(worst possible condition).

\section{VISIA imaging}

At baseline and weeks 8 and 12 post-treatment, full-face digital images were taken of each subject (right- and left-side and center views) using the VISIA photo-station (Canfield Imaging Systems, Fairfield, New Jersey) with a Canon Mark II 5D digital SLR camera (Canon Incorporated, Tokyo, Japan) under standard reproducible lighting.

\section{Subject global assessment}

Subject global assessments were performed at baseline and 8 and 12 weeks post-treatment. The cosmetic global assessment is a validated and commonly used tool to assess subject perception of their skin and give standardized feedback [14]. The questions focused on global improvement (overall condition of the skin, overall appearance of the skin, global facial fine lines and wrinkles, and overall skin tone), specific areas of improvement (skin youthfulness, forehead fine lines and wrinkles, eye area fine lines and wrinkles, skin softness and smoothness, skin elasticity, radiance, and clarity) and finally, subject satisfaction (feeling more confident in the way the skin looks, continuing to use the product, and recommending treatment to others).

\section{Subject satisfaction}

Subject satisfaction surveys were conducted at 8 and 12-week follow-ups. Subject satisfaction was graded on a 6-point scale, with a score of 1 being the least satisfied and 6 being the most satisfied.

\section{Results}

\section{Photo documentation and analysis}

All 5 subjects saw improvement in overall photoaging, including brown spots, lines, and wrinkles as early as 8 weeks (Figures 1-3).

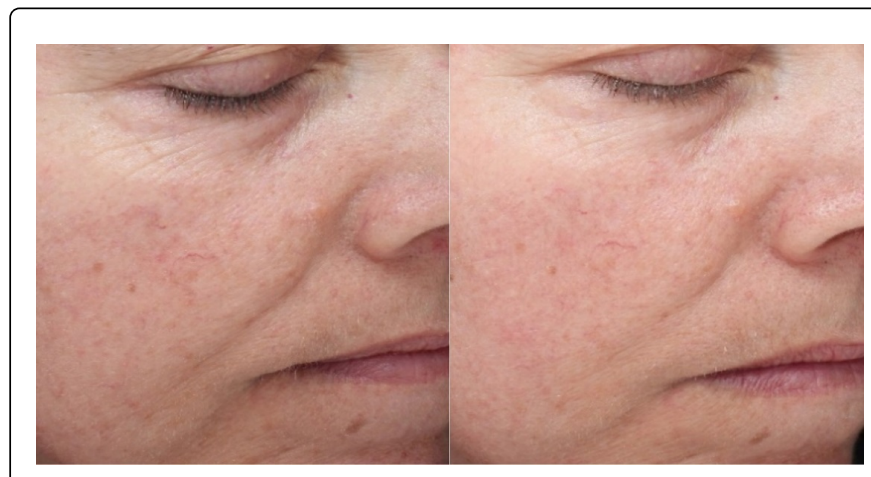

Figure 1: Clinical photographs. Compared to baseline (left), subjects treated for 8 weeks with the tripeptide/hexapeptide anti-aging regimen (right) demonstrated improvement in nasolabial fold prominence, skin texture, and redness.

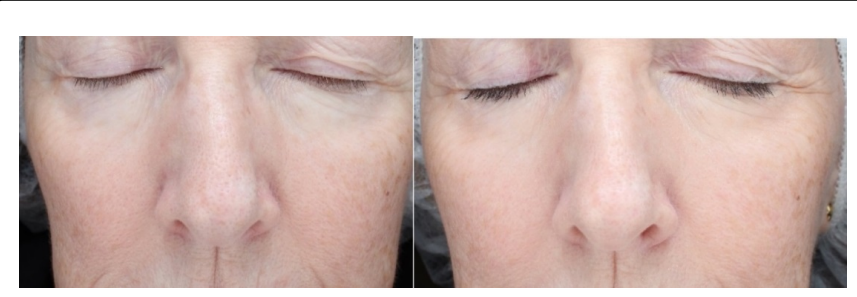

Figure 2: Clinical photographs. Compared to baseline (left), subjects treated for 8 weeks with the tripeptide/hexapeptide anti-aging regimen (right) demonstrated improvement in nasolabial fold prominence, skin texture, and redness. 


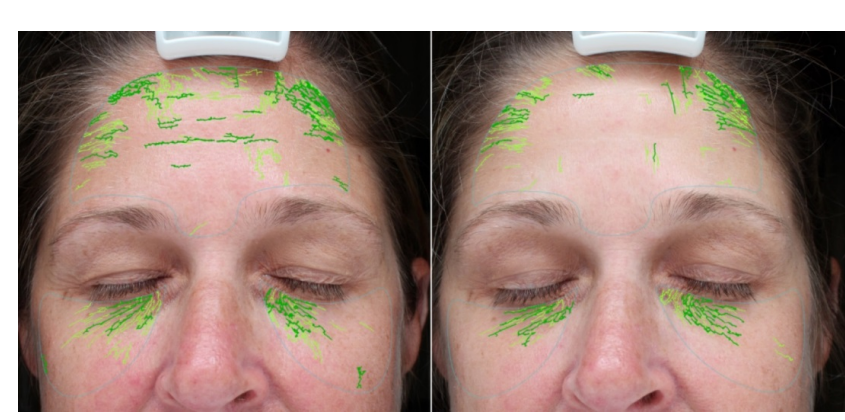

Figure 3: Visia Data analysis. Compared to baseline (left) subjects treated for 8 weeks with the tripeptide/hexapeptide anti-aging regimen (right) demonstrated reduced forehead and eyelid wrinkles in Visia images analyzed for wrinkles.

\section{Physician grading of efficacy}

Compared with baseline, all subjects improved at both 8 and 12 weeks (Figures 1-3). The most notable improvement during this study was in radiance with $44.8 \%$ mean improvement from baseline (paired sample Student's t test, $\mathrm{P}=0.004$, Figure 4). Regarding fine lines, the average percent improvement at 12 weeks was $27.7 \%$ from baseline (paired sample Student's t test, $\mathrm{P}=0.005$, Figure 4). Skin firmness and skin plumpness also showed $37.2 \%$ and $31.6 \%$ improvement, respectively (paired sample Student's $\mathrm{t}$ test, $\mathrm{P}=0.003$ and 0.03 , respectively, Figure 4). Finally, skin sagginess and wrinkle assessment both showed roughly $29 \%$ improvement from baseline (paired sample Student's $\mathrm{t}$ test, $\mathrm{P}=0.02$ for both, Figure 4 ). Based on the physician assessment, the average percent improvement for each category was $33 \%$, with radiance being the most enhanced quality.

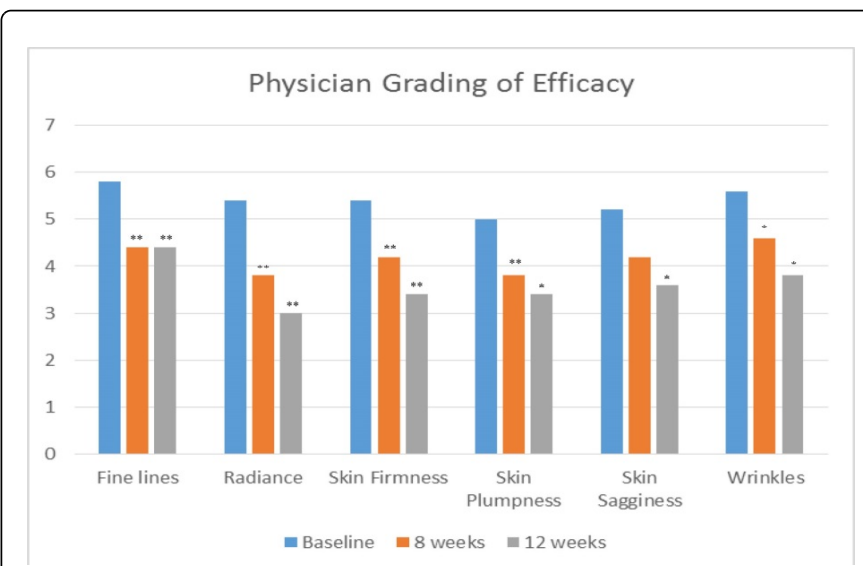

Figure 4: Physician Grading of Efficacy. Bar graphs represent the mean scores for the subjects for 6 photoaging qualities. The scale was 0-9, 0 representing the best score and 9 represented severe photoaging. The majority of mean scores improved significantly from baseline after 8 and 12 weeks of treatment with the tripeptide/ hexapeptide anti-aging regimen. ${ }^{*}$ Denotes statistical significance at $\mathrm{P}<0.05$. ${ }^{* *}$ Denotes statistical significance at $\mathrm{P}<0.01$.

\section{Subject global assessment}

After 8 weeks of use, each subject reported improvement on several of the global assessment categories (Figure 5). Subjects reported the most improvement in "my skin feels softer and smoother." All subjects agreed that they "would continue to use this product." At 8 weeks, subjects were already noticing improvement in their skin's appearance and texture.

At 12 weeks, all subjects either strongly agreed or agreed that the TriHex formulation improved their skin quality in each category assessed by the survey (Figure 5). The statement which received the most positive scoring was "improved my overall skin tone," with 4 subjects strongly agreeing with that statement.

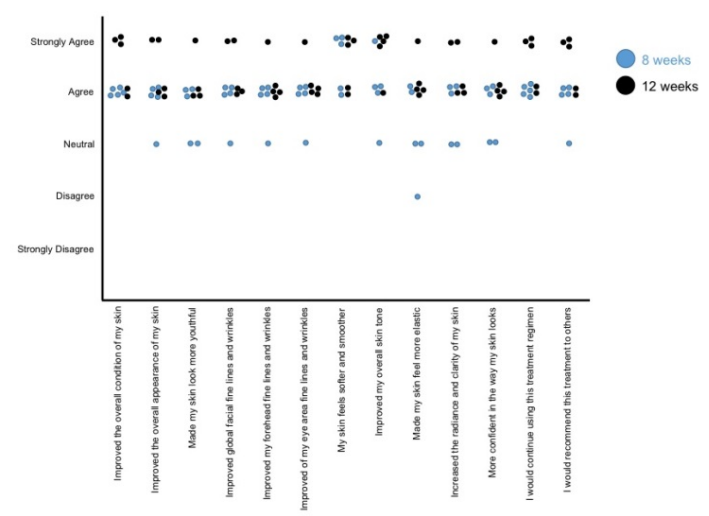

Figure 5: Subject Global Assessment Survey. A scatter plot is shown with the answer choices on the $y$-axis and the global assessment statement on the $\mathrm{x}$-axis. Each subject is represented by a circle. Eight-week data are presented by blue circles and 12-week data are presented by black circles. As plotted, there are no neutral or negative replies at 12 weeks after treatment with tripeptide/ hexapeptide anti-aging regimen, every subject scored each statement with a positive response.

\section{Subject satisfaction}

Consistent with the global assessment, all of the subjects scored their satisfaction as $5-6$ by 12 weeks.

Taken together, both physician grading and subject surveys (both global assessment and satisfaction) found that using the TriHex formulation twice a day, along with a regimen of a gentle cleanser, facial moisturizer and sunscreen improved every aspect of photoaging assessed during the study. The physician grading showed improvement by an average of $33 \%$ from baseline in fine lines, radiance, skin firmness, skin plumpness, skin sagginess, and wrinkles. Overall skin radiance was most improved, with a $44 \%$ improvement from baseline at 12 weeks. The global assessment for facial aesthetics demonstrated that subjects noticed improvement as early as 8 weeks, and by 12 weeks, all of the categories improved. Based on subject assessments, the overall skin tone was most improved. All of the subjects agreed that they would continue to use the TriHex formulation and would recommend it to others. 


\section{Discussion}

UV radiation exposure and accumulation are known causes of photoaging [2]. Appropriate skin protection with moisturizers and sunscreens are established topical regimens that help combat the effects of UV aging. UV exposure not only causes DNA damage, but it can lead to break down of the ECM of the skin [1,3]. Furthermore, many people experience increased pigmentation and/or solar lentigines, as a result of sun exposure. These brown spots, along with skin texture, wrinkles and overall tone and appearance are common complaints by dermatologic patients [15].

This investigation demonstrates the positive effects of a novel formulation with a blend of synergistic peptides and botanicals on photoaging. The TriHex formulation is one of the first topical treatments that comprehensively focuses on ECM recycling by including actives that promote both intracellular and extracellular protein rejuvenation. The robustness of these proteins is achieved by 1) clearing photo-damaged and aged proteins in the ECM and within cells, 2) controlling potentially harmful proteases, 3) regenerating collagen and elastin, 4) increasing the volume of the ECM and subdermis to enhance fibroblast function, and 5) quenching oxidation [9].

Actives in the TriHex formulation show evidence for clearing damaged proteins by stimulating matrix metalloproteinase-2, or MMP-2, in ECM (tripeptide) and the ubiquitin protease system and phagocytosis inside cells (tripeptide and phosphatidylserine) [16]. Phosphatidylserine also may decrease MMP1 and collagenase, proteases that can cause damage [17]. In the TriHex formulation, the unique combination of peptides have been shown to trigger production of collagen and elastin in the ECM [6]. Additionally, the 2 actives, hexapeptide 38 and ornithine, may potentially be PGC1 $\alpha$ stimulators which cause adipogenesis and increase volume of the ECM, and thus optimize fibroblast function. Finally, antioxidant actives, such as niacinamide and Dunaliella Salina extract, help combat DNA damage and melanin deposition and production [10-12].

\section{Conclusion}

Both subject and physician evaluations showed significant improvement in facial photoaging as early as 8 weeks after use of this tripeptide/hexapeptide anti-aging regimen. The anti-aging regimen improves multiple signs of photoaging using a synergistic blend of peptides and active botanicals.

\section{Conflict of Interest}

Dr Ortiz is a paid consultant to Alastin SkinCare Inc (Carlsbad, CA)

\section{References}

1. Fisher GJ, Kang S, Varani J, Bata-Csorgo Z, Wan Y, et al. (2002) Mechanisms of photoaging and chronological skin aging. Arch Dermatol 138: $1462-1470$
2. Gragnani A, Cornick S, Chominski V, Ribeiro de Noronha S, Alves Corrêa de Noronha S, et al. (2014) Review of Major Theories of Skin Aging. Adv Aging Res 3: 265-284.

3. Hwang Ka, Yi BR, Choi KC (2011) Molecular mechanisms and in vivo mouse models of skin aging associated with dermal matrix alterations. Lab Anim Res 27: 1-8.

4. Wu X, Hammer JA (2014) Melanosome transfer: it is best to give and receive. Curr Opin Cell Biol 29: 1-7.

5. Badenhorst T, Svirskis D, Merrilees M, Bolke L, Wu Z (2016) Effects of GHK-Cu on MMP and TIMP Expression, Collagen and Elastin Production, and Facial Wrinkle Parameters. J Aging Sci 4: 2-7.

6. Widgerow AD, Fabi SG, Palestine RF, Rivkin A, Ortiz A, et al. (2016) Extracellular Matrix Modulation: Optimizing Skin Care and Rejuvenation Procedures. J Drugs Dermatol 15: S63-S71.

7. Siméon A, Wegrowski Y, Bontemps Y, Maquart FX (2000) Expression of Glycosaminoglycans and Small Proteoglycans in Wounds: Modulation by the Tripeptide-Copper Complex Glycyl-L-Histidyl-L-Lysine-Cu2+. J Invest Dermatol 115: 962-968.

8. Puigserver P (2005) Tissue-specific regulation of metabolic pathways through the transcriptional coactivator PGC-1a. Int J Obes 29: Suppl $1:$ S5-S9.

9. Widgerow AD, Jiang LI, Calame A (2018) A single-center clinical trial to evaluate the efficacy of a tripeptide/hexapeptide antiaging regimen. J Cosmet Dermatol

10. Hemalatha A, Girija K, Parthiban C, Saranya C, Anantharaman P (2013) Antioxidant properties and total phenolic content of a marine diatom, Navicula clavata and green microalgae, Chlorella marina and Dunaliella salina. Adv. Appl. Sci. Res 4: 151-157.

11. Vílchez C, Forján E, Cuaresma M, Bédmar F, Garbayo I, et al. (2011) Marine Carotenoids: Biological Functions and Commercial Applications. Mar. Drugs 9: 319-333.

12. Wertz K, Hunziker PB, Seifert N, Riss G, Neeb M, et al. (2005) R: betaCarotene interferes with ultraviolet light A-induced gene expression by multiple pathways. J Invest Dermatol 124: 428-434.

13. Stangroom J. Pairedsample Student's $t$ test. Social Science Statistics website. http://www.socscistatistics.com/tests/ttestdependent/ Default.aspx.

14. Birnbaum JE, McDaniel DH, Hickman J, Dispensa L, Le Moigne A, et al. (2017) A multicenter, placebo-controlled, double-blind clinical trial assessing the effects of a multicomponent nutritional supplement for treating photoaged skin in healthy women. J Cosmet Dermatol 16: 120-131.

15. Helfrich YR, Sachs DL, Voorhees JJ (2008) Overview of skin aging and photoaging. Dermatol Nurs 20: 177-183.

16. Pickart L, Vasquez-Soltero JM, Margolina A (2014) GHK and DNA: Resetting the Human Genome to Health. BioMed Research International 2014:1-10

17. Lee SH, Yang JH, Park YK, Han J, Chung G, et al. (2013) Protective effect and mechanism of phosphatidylserine in UVB-induced human dermal fibroblasts. European J Lipid Sci Tech 115: 783-790. 